\title{
Erythrocyte phagocytosis in the human trabecular meshwork
}

\author{
IAN GRIERSON AND WILLIAM R. LEE \\ Tennent Institute of Ophthalmology, University of Glasgow
}

The mechanisms of erythrocyte disposal by the trabecular meshwork in the human eye have not been extensively studied by electron microscopy. At the present time there is one short report on the ultrastructural changes induced by post-traumatic recurrent hyphaemas in two elderly patients. In both patients, Yamashita and Rosen (1965) observed infiltration of the endothelial meshwork by phagocytic endothelial cells and debris and they suggested that the transient glaucoma, which was observed clinically, was due to a mechanical obstruction. In order to add more information with regard to the pathogenesis of haemolytic glaucoma (Fenton and Zimmerman, 1963), the following report describes the changes induced in the trabecular meshwork of a 20-year-old man 2 weeks after recurrent bleeding into the anterior chamber.

\section{Case report}

The right eye of a 20-year-old man was perforated by a metal splinter which passed through the inferior limbus, tore the inferior iris, and remained in the inferior angle. The following day the fragment was removed by an electro-magnet, prolapsed iris tissue was excised, and the limbal wound sutured. Subsequently, the ocular tension remained normal, but intraocular haemorrhage continued intermittently and the blind painful eye was enucleated I 5 days after the original injury.

\section{Pathology}

\section{Materials and methods}

The enucleated globe was immersed in 3 per cent. glutaraldehyde (buffered to $\mathrm{pH} 7 \cdot 3$ by cacodylate) after a trephine hole had been made in the central cornea and the anterior chamber gently irrigated by pressure on the posterior segment. The globe was opened by a parasagittal section and the 'pupil-optic nerve' block was processed for paraffin histology. Tissue from the angles was obtained from the calottes and processed for electron microscopy by post-fixation in I per cent. osmium tetroxide, dehydration in graded alcohols, and passage through propylene oxide before being embedded in Araldite. The tissue was sectioned on an LKB Ultratome I I ; thick sections were stained with toluidine blue and ultrasections $(500-800 \AA)$ were stained with uranyl acetate and lead citrate for examination in a Seimens Elmiskop I A.

\section{Gross examination}

There was a sutured perforating wound at the inferior limbus, a traumatic peripheral iridectomy, and prolapse of the inferior part of the ciliary body into the wound. The anterior chamber and the inferior vitreous contained blood, the posterior retina was detached by blood, and the choroid was thickened by foci of recent haemorrhage. 


\section{Light microscopy}

Microscopic examination revealed the source of haemorrhage to be the result of a tear in the iris stroma. In paraffin sections, the trabecular meshwork contained numerous red cells, and iron-containing macrophages were present in the angle and the intertrabecular spaces. In Araldite-embedded I $\mu$-thick sections, the most obvious feature was infiltration of the trabecular meshwork by a polymorphic cellular infiltrate which included erythrocytes, lymphocytes, plasma cells, polymorphonuclear leucocytes, mast cells, histiocytes, and giant macrophages (Fig. I). Giant macrophages (up to $40 \mu$ diameter) containing lipid vacuoles and ingested erythrocytes were present on the anterior surface of the iris, theinner surface of the meshwork, and the inner layers of the uveal meshwork (Fig. I).

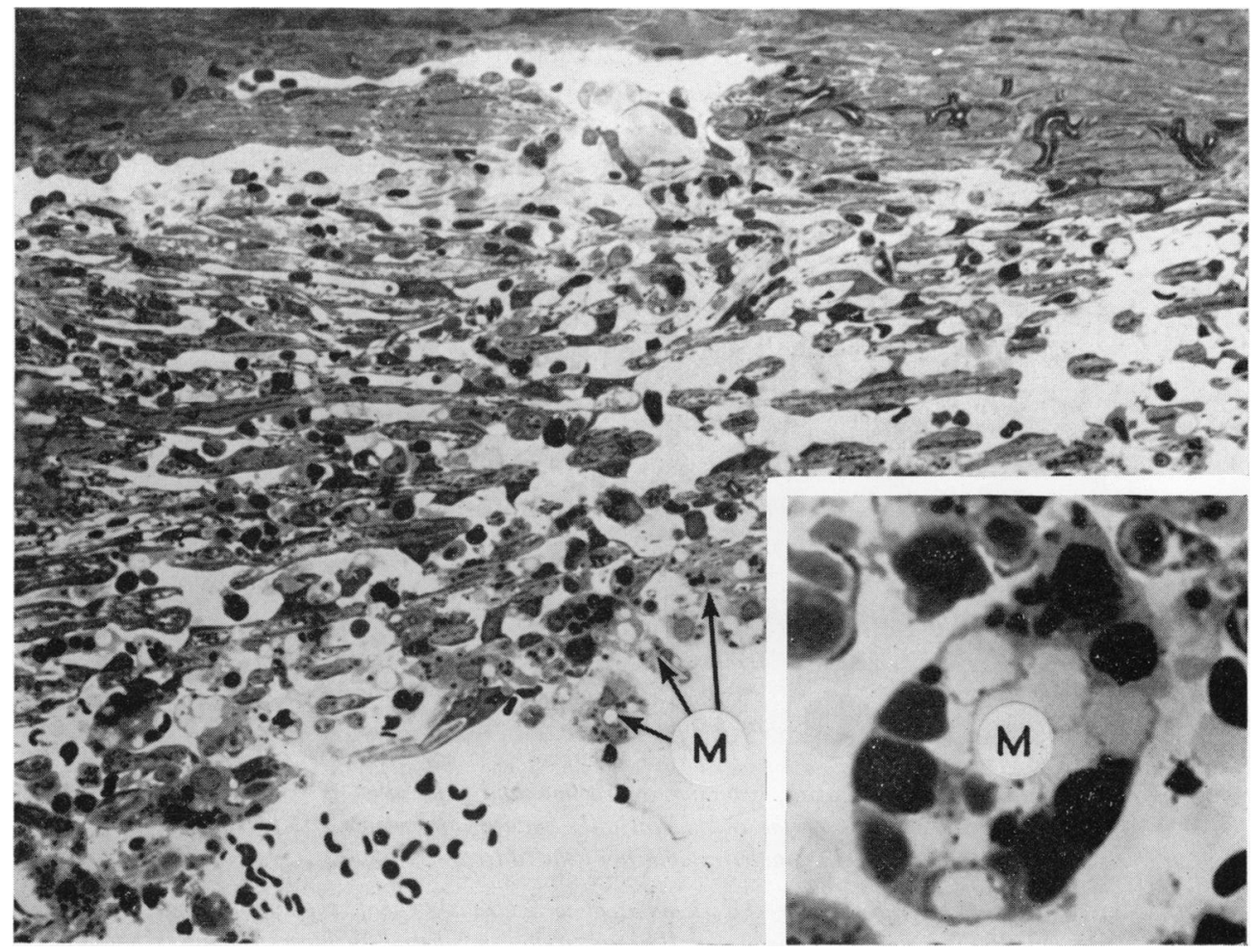

FIG. I Trabecular meshwork after a 2-week hyphaema, showing giant macrophages (M), swollen endothelial cells, and histiocytes in the intertrabecular spaces $\left(\times 35^{\circ}\right)$. Insert shows a giant macrophage containing engulfed erythrocytes, cell debris, and clear vacuoles $(\times \mathrm{I}, 430)$. Toluidine blue-stained $\mathrm{I} \mu$-thick section

Passage of these cells beyond the inner uveal meshwork appeared to be limited mechanically by the diminishing size of the orifices in the uveal trabecular beams. These beams were distorted and disrupted and appeared to have been damaged by mechanical pressure. Endothelial cells in this region and in the corneo-scleral meshwork were diminished in number and were contracted and rounded, so that the cytoplasm covering the trabecular cores was attenuated and the surfaces of many trabecular cores were denuded. Some of the endothelial cells contained intracytoplasmic erythrocytes and cell debris and their darker-staining cytoplasm distinguished them from the numerous phagocytic histiocytes 
which had a paler cytoplasm (Fig. 2a). Although the distinction between these two types of cell was often easy, there were also "intermediate" forms which could not be classified with certainty. A few phagocytes containing melanin were present in the uveal meshwork.

The structure of the endothelial (juxtacanalicular) meshwork was better preserved, and even though there were numerous erythrocytes and macrophages in the spaces between the cells, the subendothelial connective tissue was of a loose texture and the endothelial cells were of relatively normal appearance.

The endothelial lining of the trabecular side of Schlemm's canal was abnormally thin, but was in continuity. Vacuolated endothelial cells were numerous, but in most instances the vacuoles appeared empty although a few contained red cells. Migratory phagocytes were occasionally seen to be entrapped in the lining endothelium, but whether this was through a damaged cell, between loosened cell junctions, or via intracellular pores could not be determined at this level of magnification (Fig. $2 b$ ).
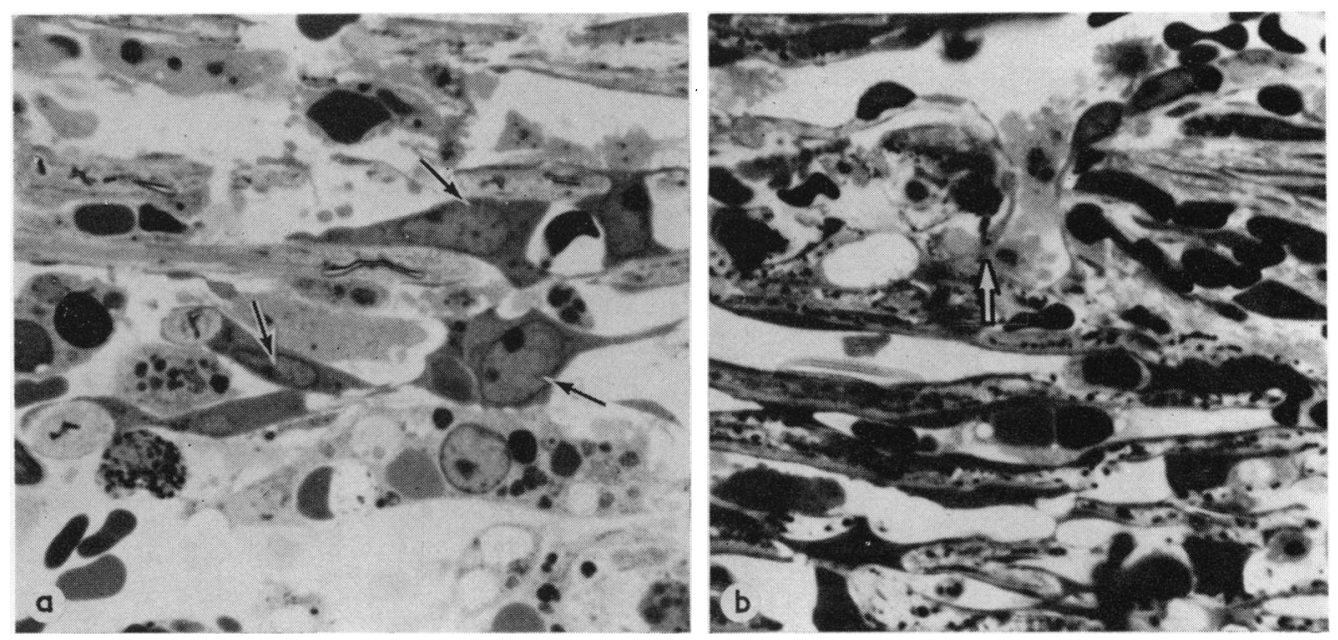

FIG. $2 a$ Uveal meshwork, showing contraction and separation of endothelial cells (arrows) to expose the trabecular cores. Some of the endothelial cells contain intracytoplasmic particles and can be distinguished from phagocytic histiocytes by the paler cytoplasm of the latter

FIG. $2 b$ The trabecular meshwork adjacent to Schlemm's canal, showing a migratory phagocyte entrapped in the endothelial lining layer (arrow). The underlying endothelial meshwork contains erythrocytes and the connective tissue is of a loose texture. Toluidine blue-stained $\mathrm{I} \mu$-thick sections. $\quad \times 950$

\section{Electron microscopy}

In many respects the architecture of the meshwork was undisturbed in spite of prolonged exposure to large numbers of erythrocytes. Some of the intertrabecular spaces were empty, but many contained erythrocytes and other haematogenous cells. The erythrocytes in the intertrabecular spaces were of variable appearance; some were intact, but many were depleted of haemoglobin and the cytoplasm consisted of condensed clumps of coarse osmiophilic granules lying within a finely granular flocculent material (Fig. 3). Whether or not extracellular digestion is a feature of this system we cannot say, but the presence in the intertrabecular spaces of cellular profiles, red cell fragments, and erythrocytes with a damaged plasma membrane makes it a possibility to be considered. Although 
some endothelial cells maintained their normal relationship to the trabecular core, others were lying free in the intertrabecular spaces or were apparently in the process of detachment from the trabecular beams (Fig. 3). This endothelial stripping was particularly prominent in the uveal meshwork, where long segments of the beams were denuded. The collagen core of the denuded beam was wider than normal and the periphery was disrupted so that the collagen and basement membrane fragments were scattered (Fig. 3). In some, but not all, of the trabecular beams in which there was endothelial detachment, multilayering of the basement membrane was observed (Fig. 3).

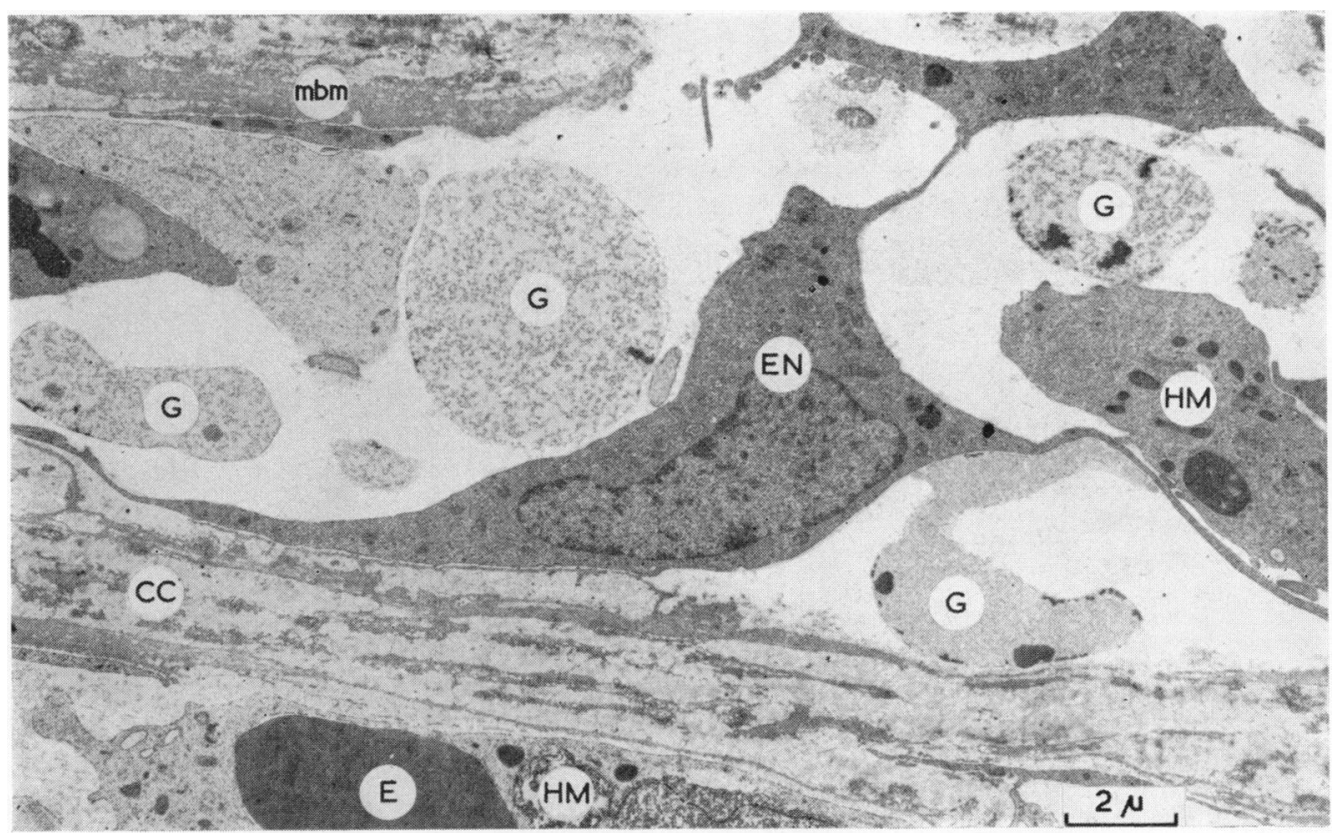

FIG. 3 The corneo-scleral meshwork, showing erythrocyte ghosts $(G)$ and histiocytic macrophages $(H M)$ in the intertrabecular spaces; one of these histiocytic macrophages contains an erythrocyte fragment $(E) . A n$ endothelial cell $(E \mathcal{N})$ is partially detached from the degenerate trabecular collagen core $(C C)$. The basement membrane $(\mathrm{mbm})$ of the beam at the upper left of the figure is multilayered. $\times 5,000$

Endothelial separation was associated with distinct ultrastructural changes. In the initial stage, micropinocytosis was diminished and lysosomes were seen within the cytoplasm (Fig. 4). With advancing detachment, micropinocytosis was markedly diminished and intracytoplasmic filaments were reduced in number (Fig. 3). At the end-stage the cell membrane was smooth and the cytoplasm contained numerous mitochondria and lysosomes; intracytoplasmic filaments were absent. Rough endoplasmic reticulum was prominent and evenly distributed throughout the cytoplasm (Fig. 5). The detaching and detached endothelial cells were on occasion in close proximity to erythrocytes and the cytoplasmic processes from one or more endothelial cells extended around the erythrocyte (Fig. 5).

Phagocytosis of erythrocyte fragments and breakdown products was observed in detaching and detached endothelial cells (Fig. 6), and since these cells could be distinguished from wandering histiocytic macrophages they will be referred to as endothelial macrophages in the following text. 


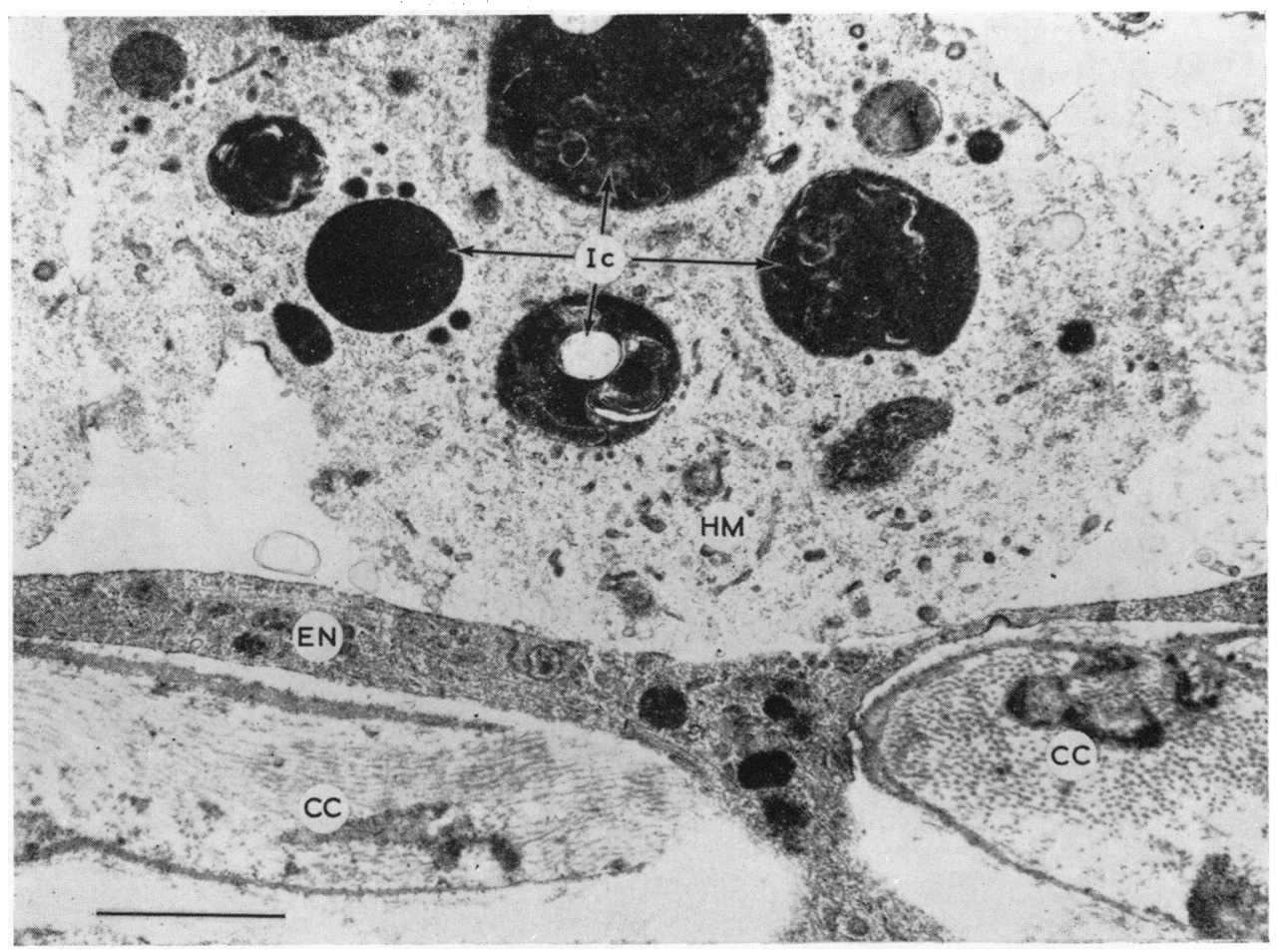

FIG. 4 Large histiocytic macrophage $(H M)$, resting on an endothelial cell in the cornco-scleral meshwork. The macrophage contains several lysosomal complexes $(l c)$ which probably represent stages in erythrocyte breakdown; the cytoplasmic rarefaction is a feature associated with advanced phagocytic activity. The endothelial cell $(E N)$ lining the trabecular core $(C C)$ contains lysosomes and shows reduced micropinocytotic activity. $\quad \times 16,750$

(i) The cytoplasm of these endothelial macrophages did not differ in any significant way from that of the detached endothelial cells described above.

(ii) Histiocytic macrophages had the following ultrastructural characteristics and conformed to the description provided by Weiss (1972). These cells had ovoid or indented nuclei (Fig. 2a) and the cytoplasm contained numerous mitochondria and lysosomes, large lipid vacuoles, small pseudopodia or microvilli, and abundant free ribosomes (Figs 3, 4, Io, and I I). In advanced phagocytosis, cytoplasmic rarefaction was a notable feature in histiocytic macrophages (Fig. 4), some of which assumed the form of giant macro. phages and exhibited a prolific form of multiphagocytosis.

The phagocytosed material in both histiocytic and endothelial macrophages was of variable appearance. In some cells large homogeneous erythrocyte fragments were present in the cytoplasm, while in others a manifestation of erythrocyte breakdown was the formation of heterolysosomes or lysosomal complexes; the latter have both intrinsic (Golgi-produced lysosomes) and extrinsic (phagocytosed material) components. These were multilayered electron dense bodies of variable shape and size, but of the order of $\mathrm{I} \mu$ in diameter (Figs 4,9 , and io).

In another form of degradation the cytoplasm of the macrophages contained large bodies (up to $2 \mu$ in diameter) with a granular appearance and an indistinct membrane (Fig. 7); these were considered to be accumulations of haemosiderin and were named haemosiderin bodies. 


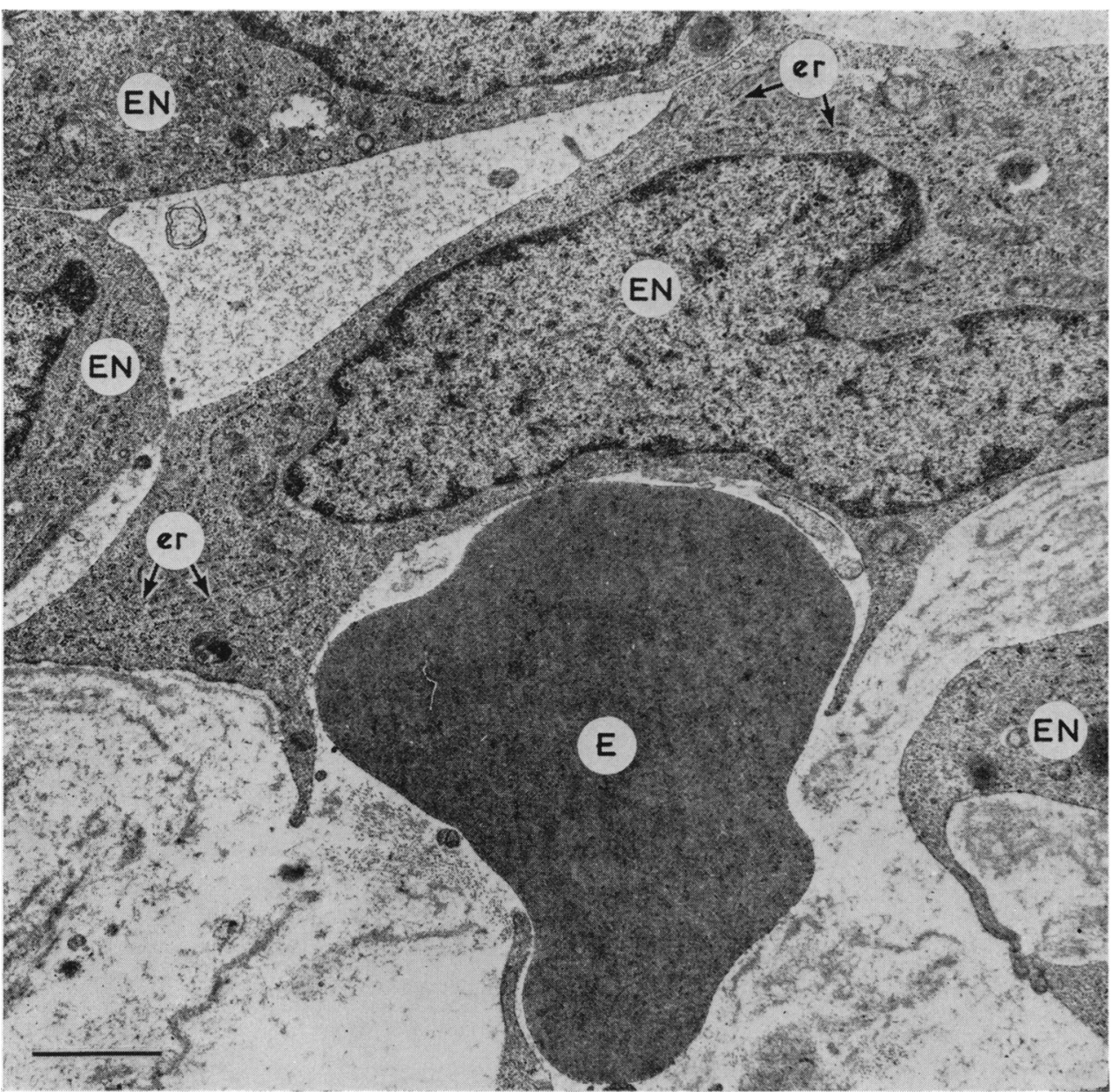

FIG. 5 Erythrocyte $(E)$ partially enmeshed by processes from detached endothelial cells $(E \mathcal{N})$. The cytoplasm of the endothelial cells contains large quantities of rough endoplasmic reticulum $(\mathrm{er}) . \quad \times \mathrm{I}_{5}$,000

The initial qualitative impression was that there were many more free erythrocytes in the outer, or endothelial, meshwork than in the uveal meshwork. To confirm this, a quantitative assessment was made by counting free and phagocytosed erythrocytes in the three anatomical regions of the meshwork in random grids from different blocks. In the endothelial meshwork, 58 I erythrocytes were counted and of these 72 were ingested (i.e. approximately $\mathrm{I} 2$ per cent. were phagocytosed). In the corneo-scleral meshwork, 687 erythrocytes were counted and of these 163 were ingested (i.e. approximately 24 per cent. were phagocytosed). In the uveal meshwork, 736 erythrocytes were counted and of these 572 (i.e. approximately 78 per cent.) were phagocytosed. These data suggest, therefore, that erythrocyte phagocytosis was more active in the inner layers of the meshwork.

Free endothelial cells were sparse in the uveal meshwork and endothelial macrophages had accumulated in the outer meshwork, which implies either that the detached endothelial cells had migrated actively or had been passively washed through the meshwork. Although 


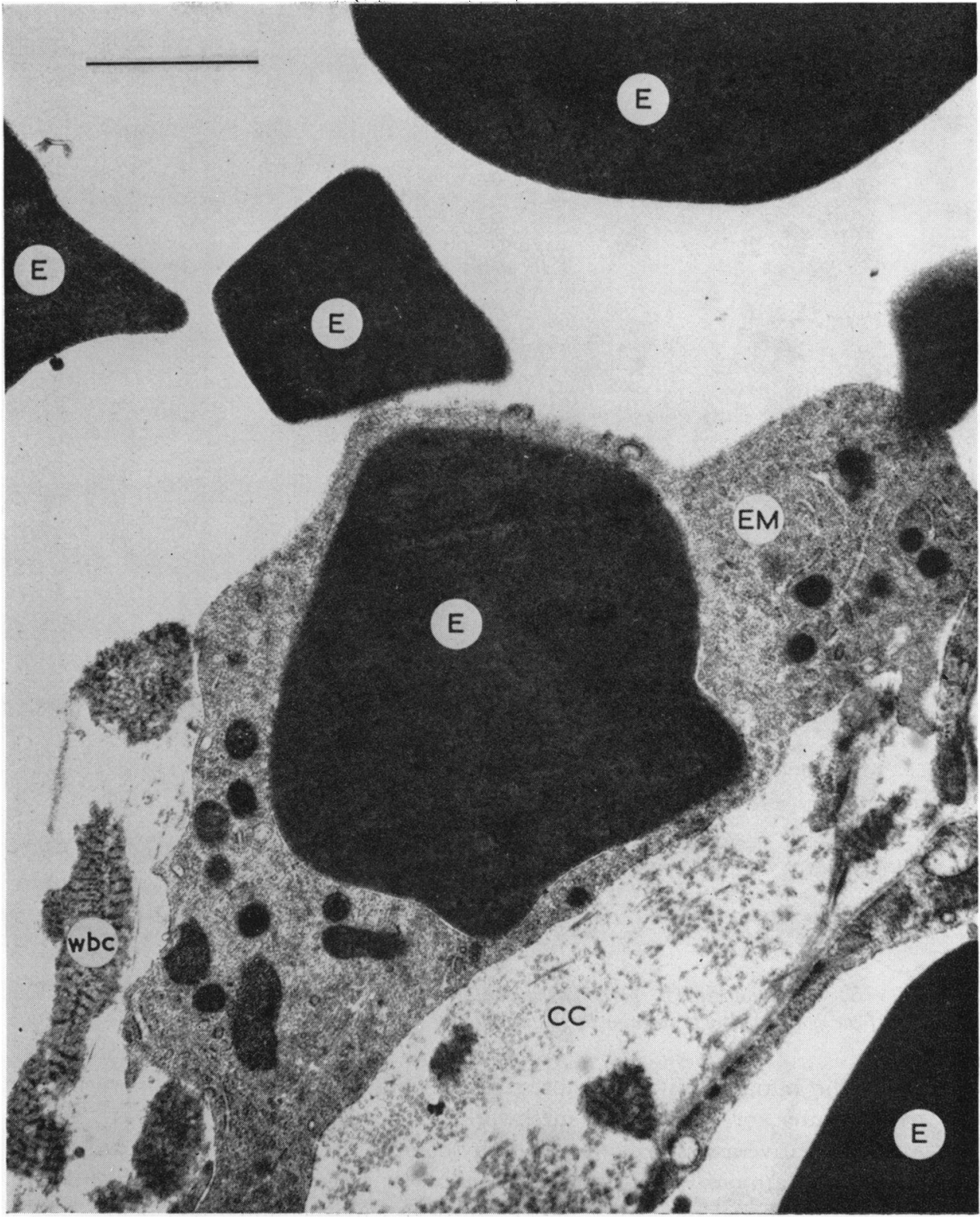

FIG. 6 Trabecular endothelial macrophage $(E M)$, containing a large erythrocyte fragment $(E)$, in the process of detachment from the trabecular collagen core $(C C)$. Fragments of wide-banded collagen $(w b c)$ are lying free in the intertrabecular space. $\times 22,500$

the endothelial or juxtacanalicular meshwork contained an excess of macrophages and erythrocytes, the native cells showed remarkably little alteration at the ultrastructural level (Fig. 8). One interesting abnormality was noted, however, in the connective tissue beneath the lining endothelium. Here there were focal aggregates of small electron dense bodies about $0 \cdot 1 \mu$ in diameter; the exact nature of these particles was not resolved but it was assumed that they were modified products of erythrocyte debris. 


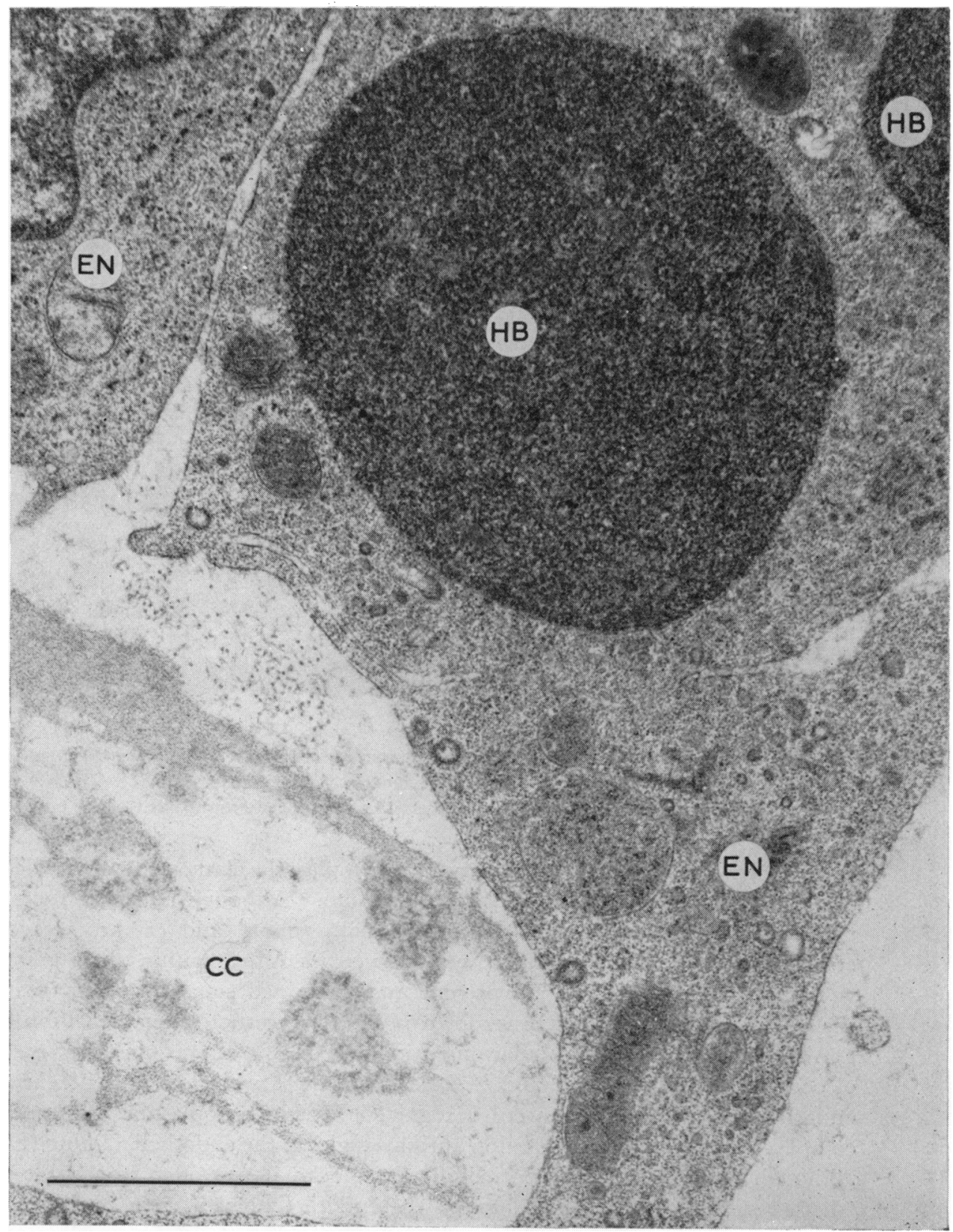

FIG. 7 The cytoplasm of many endothelial cells $(E \mathcal{N})$ was found to contain large circular masses of electron dense granular material surrounded by an indistinct membrane; these were considered to be accumulations of haemosiderin $(H B) . \quad \times 35,000$

In many areas the endothelium lining the trabecular wall of Schlemm's canal showed no ultrastructural evidence of degeneration or altered function (Fig. 8). The tight junctions were intact and the appearances were similar to those described in the normal human meshwork by Holmberg (1965), Vegge (1967), and Tripathi (1969). The cells 


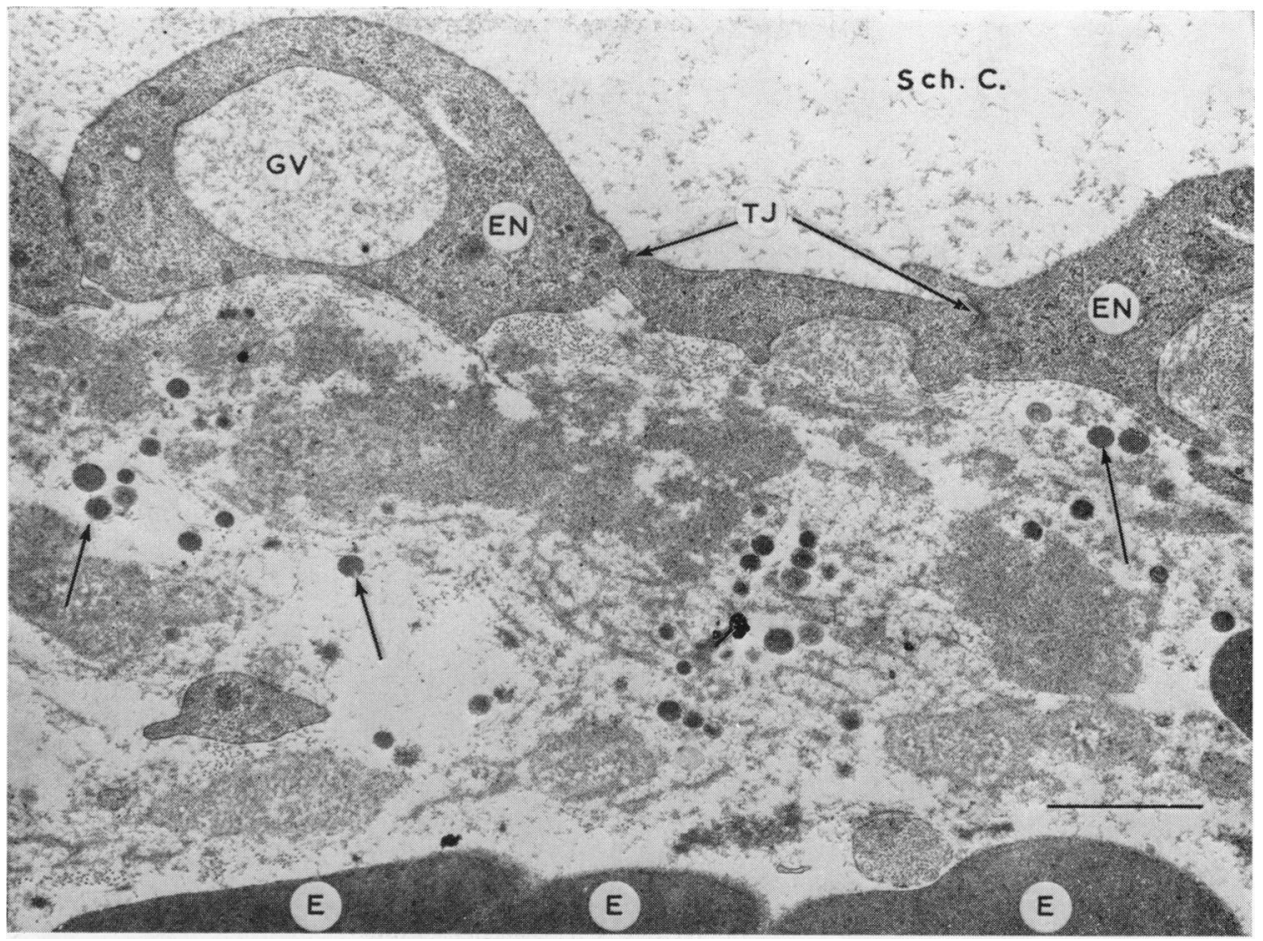

FIG. 8 Normal appearance of trabecular endothelial lining cell layer $(E \mathcal{N})$ of Schlemm's canal (Sch. C). The tight junctions $(T \mathcal{F})$ are intact and a giant vacuole $(G V)$ containing flocculent material is present in the endothelium. The underlying ground substance contains electron dense particles of an indeterminate nature (arrows) and the edges of erythrocytes $(E)$ are present within the connective tissue. $\times 15,000$

contained a few mitochondria, many intracytoplasmic filaments, a small Golgi apparatus and a normal amount of endoplasmic reticulum. Although pinocytosis was in evidence, the pinocytotic vesicles were electron-optically empty and erythrocytes or individual erythrocyte breakdown products were never identified within these micropinocytotic vesicles. Giant vacuoles were present and contained some flocculent material; whole erythrocytes or erythrocyte breakdown products were not observed within the vacuoles.

Although inter- or transcellular migration of erythrocytes was not observed, on a number of occasions both endothelial and histiocytic macrophages were found to be entrapped in the endothelium. These cells were recognized by intracytoplasmic inclusions (lysosomal complexes) and by their orientation at right-angles to the endothelial lining (Figs 9 and ro). Although these cells were seen in different blocks, it was often impossible to determine whether or not their passage through the endothelium was intracellular or intercellular (i.e. by separation of the cell junctions) because the edges of the adjacent endothelial cells were indistinct (Fig. 9). On one occasion, a histiocytic macrophage was included in serial sections and these clearly demonstrated the transmission to be via an intracellular pore (Figs Io $a$ and $b$ ) which is not part of a giant vacuole. Another morphological feature of note was the presence of focal breaks in the cytoplasm of the endothelial lining of Schlemm's canal (Fig. I I), and aggregates of degranulated blood platelets were fused with the cytoplasm of this damaged endothelium. Occasionally, larger deficits were present in the lining endothelium and these were plugged by endothelial cells, cell 


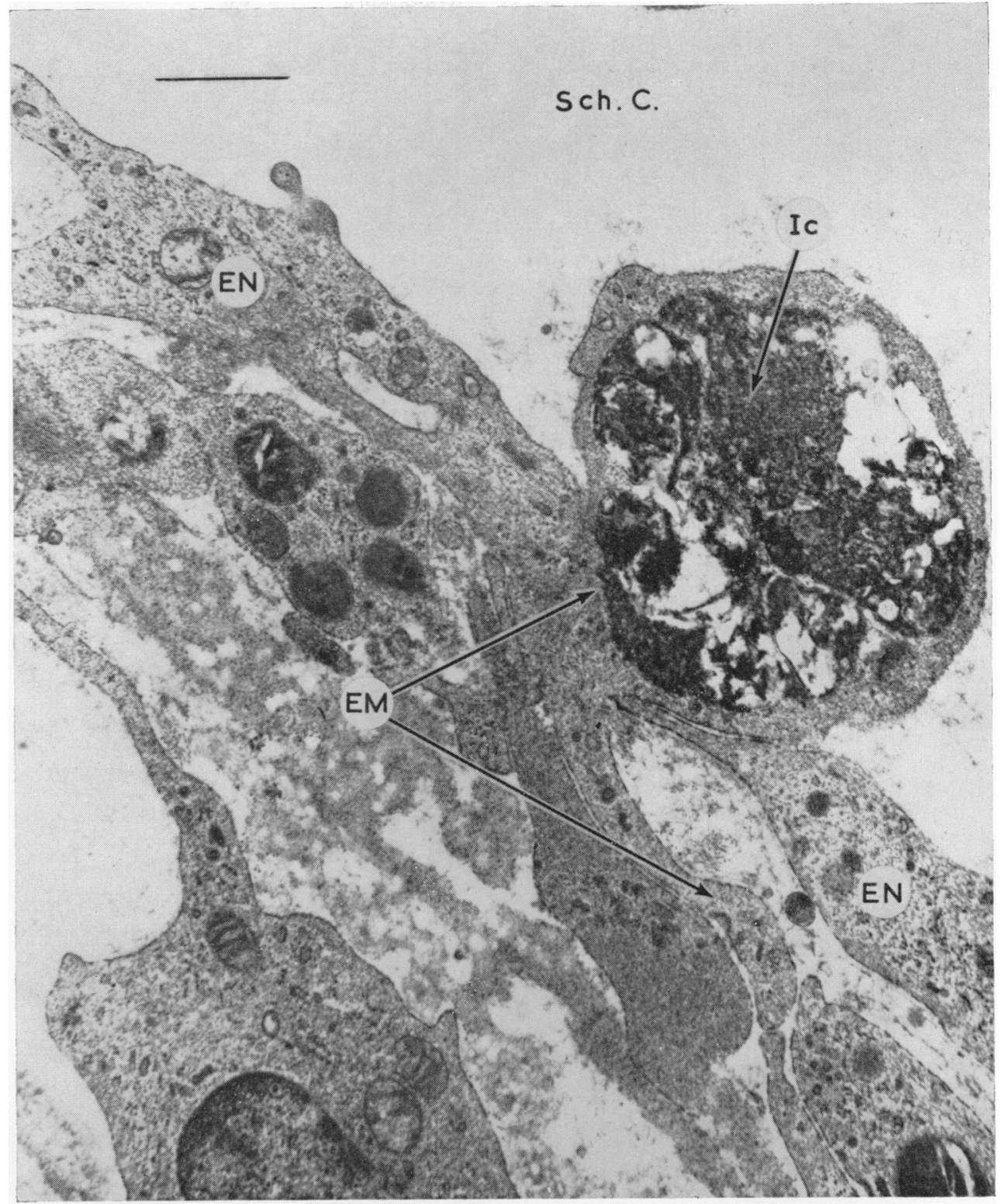

FIG. 9 Endothelium lining the trabecular wall of Schlemm's canal. Two normal endothelial cells $(E \mathcal{N})$ are separated by an endothelial macrophage (EM) which is not native to this layer. It contains a large heterolysosomal complex $(l c)$ and is probably a phagocyte from the deeper meshwork. Whether it is moving through an inter- or an intracellular passage is not evident. $\times 22,000$

debris, and platelets. Erythrocytes were often present on either side of the ruptured strip of endothelium, but on no occasion were these cells seen within the damaged area.

\section{Discussion}

The electron microscope has provided valuable information concerning the complexity of 


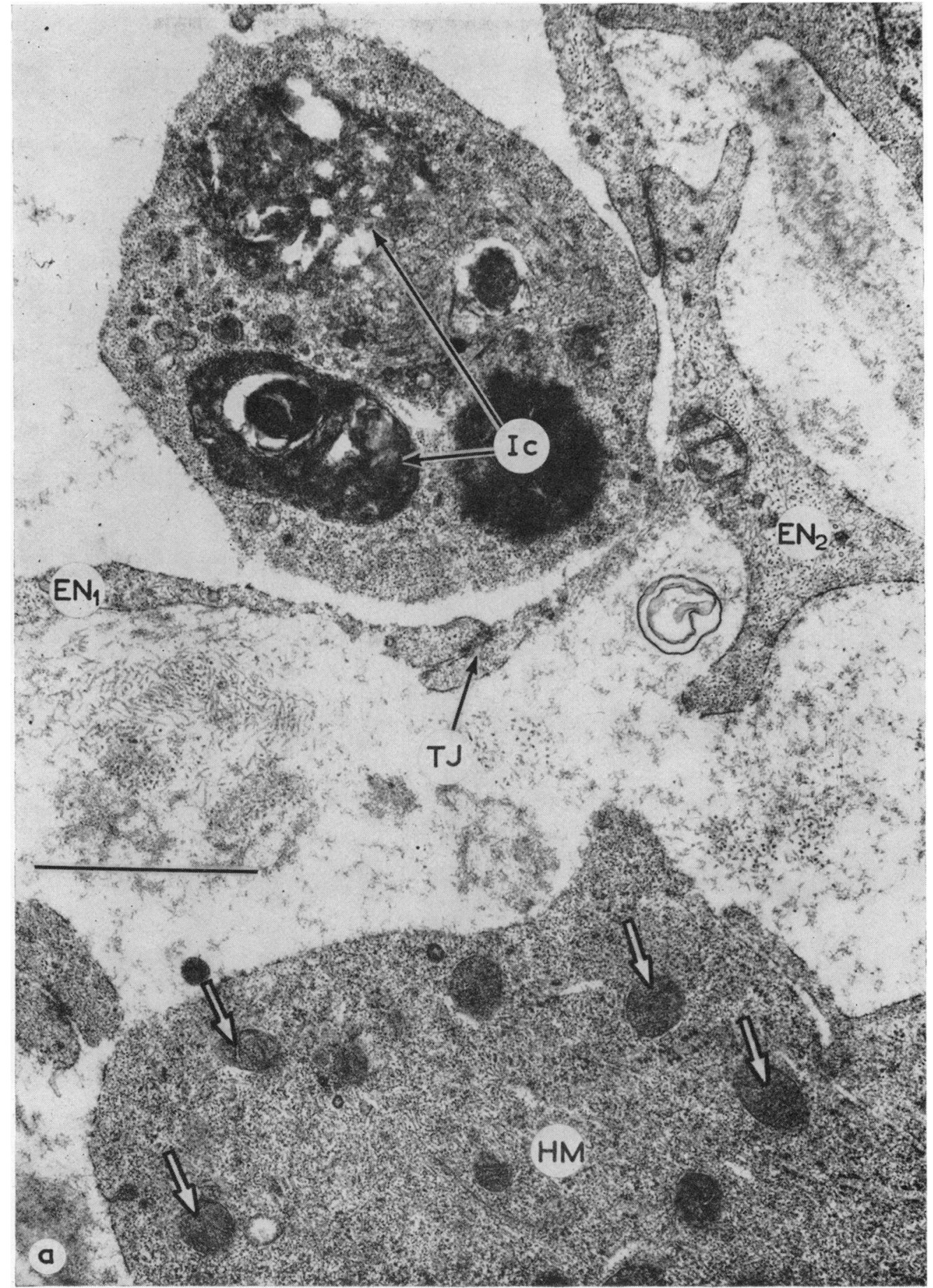

FIG. Io Serial sections demonstrate transcellular migration of a histiocytic macrophage through the endothelium lining Schlemm's canal

(10a) The endothelial cell $E \mathcal{N}_{2}$ is connected to the endothelial cell EN 1 by a tight junction (T7). The histiocytic macrophage is in two parts, one within the endothelial meshwork, the other in Schlemm's canal 


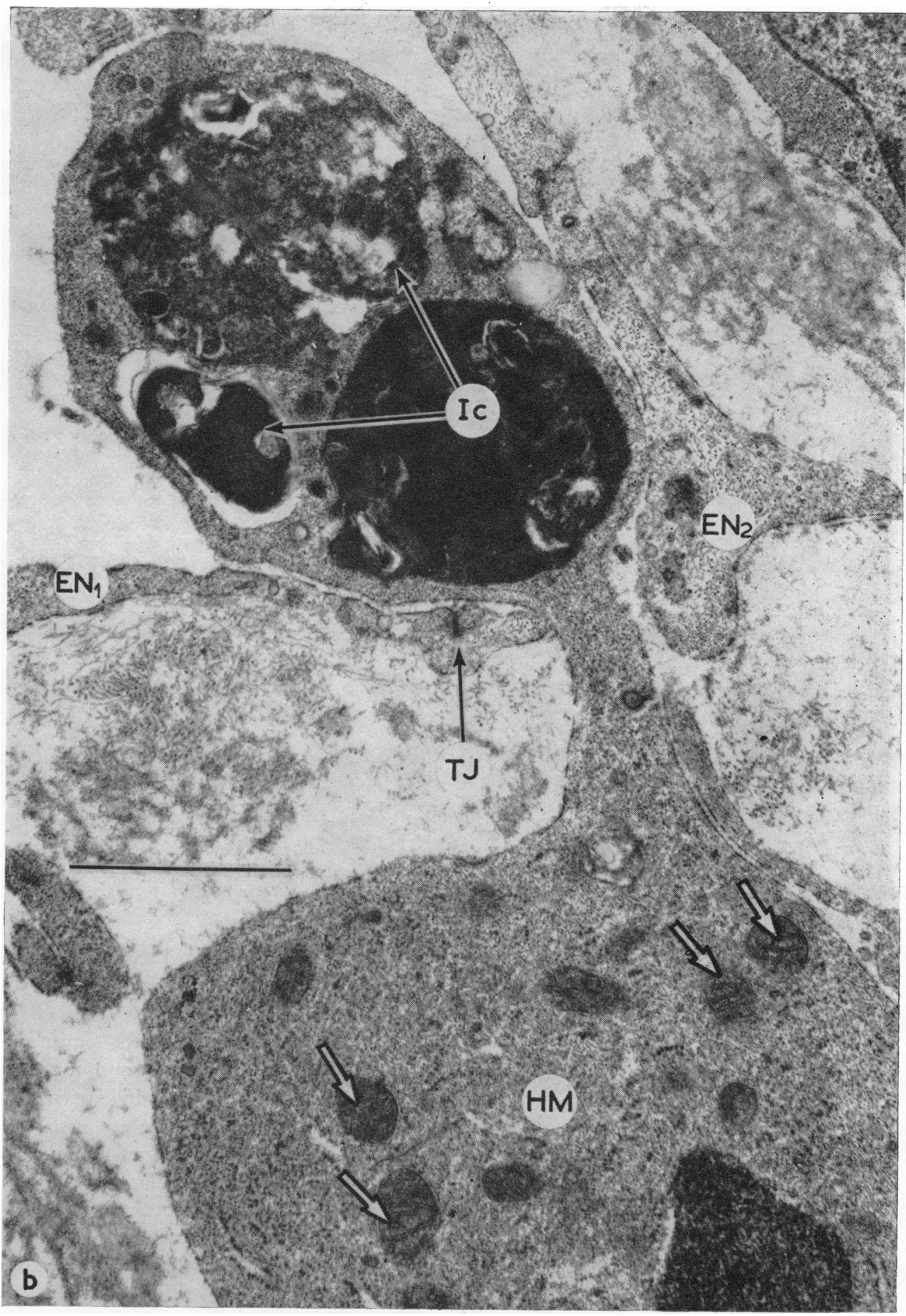

$(\mathrm{I} \mathrm{O} b)$ The two parts of the cell are connected by a narrow strip of cytoplasm which passes through a pore in the lining endothelium of the canal. The tight junction $(\mathcal{T})$ between $E \mathcal{N}_{1}$ and $E \mathcal{N}_{2}$ is intact, so that the passage must be transcellular. Note the lysosomal complexes $(l c)$ and the numerous mitochondria (arrows). $\times 33,000$ 


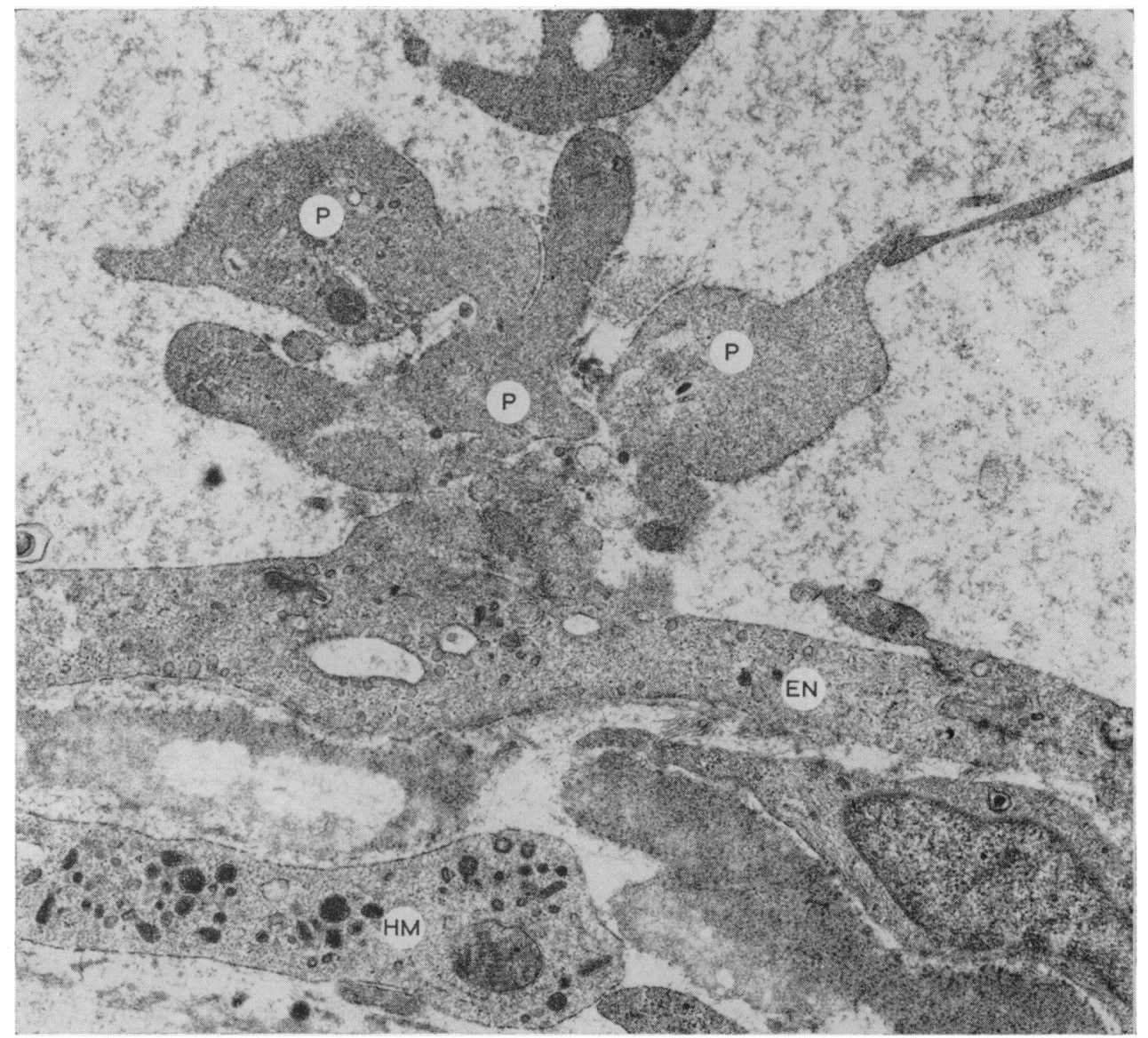

FIG. I I Small platelet thrombus on damaged endothelial lining layer. The platelets $(P)$ are degranulated and swollen and have fused with the cytoplasm extruded from the damaged endothelial cell $(E \mathcal{N}) . \quad$ Part of a histiocytico. macrophage $(H M)$ is present beneath the lining endothelium. $\times 15,000$

the cellular mechanisms involved in erythrocyte disposal. In this study, the prominent mechanism in erythrocyte clearance was found to be phagocytosis by macrophages derivedo from the endothelium and from tissue histiocytes.

The phenomenon of phagocytosis of a wide variety of particles-natural and artificial $\stackrel{D}{=}$ -by endothelial and histiocytic macrophages has been the subject of a number of investi- N gations, although frequently it has been observed coincidentally in experiments designed ton study the pathways of aqueous flow through the trabecular apparatus. MelaninN phagosomes were noted in the endothelial cells of the normal human meshwork byo Tripathi (1972) and a similar, but more advanced, melanin phagocytosis was thought too be the cause of glaucoma in the pigment dispersion phenomenon (Iwamoto, Witmer, and Landolt, I97I). Phagocytosis of exfoliated material by endothelial cells was observed ${ }^{+}$ by Ringvold and Vegge (197I) in their study of the trabecular meshwork of patients ${ }_{0}^{0}$ suffering from the syndrome of pseudoexfoliation of the lens capsule; these authors were $\stackrel{\mathbb{\Omega}}{\mathbb{\Phi}}$ able to relate the degree of meshwork saturation to the abnormal increase in intraocular $\stackrel{\overrightarrow{\mathbb{Q}}}{\mathbb{Q}}$ pressure. 
The phenomenon of microphagocytosis was studied in detail in vervet monkeys by Rohen and van der Zypen (1968), who demonstrated microphagocytosis of India ink and colloidal gold particles by trabecular endothelial cells. These authors also drew attention not only to the involvement of endothelial cells in phagocytosis, but also to their separation from the trabecular beams and their migration through the meshwork. They provided convincing evidence that macrophages can migrate through pores in the endothelium lining the trabecular wall of Schlemm's canal and, incidentally, observed macrophagocytosis of an erythrocyte by an endothelial cell. The present investigation has shown that a similar process of macrophage migration occurs in the human trabecular meshwork, and the ultrastructural characteristics of the migrating cells allow them to be classified according to their original cell type, i.e. endothelial or histiocytic. It is difficult to be certain of the site of origin of the numerous histiocytic macrophages which accounted for the major part of phagocytic activity in the tissue. These cells are normally present in small quantities in the human and monkey trabecular meshwork, but in the pathological state the additional cells could have been derived from multiplying fixed-tissue histiocytes, blood monocytes, or migratory cells from the iris or ciliary body.

The initial stage ( 1 to 8 days) of phagocytosis of erythrocytes by macrophages has been studied in the trabecular meshwork of monkeys by Shabo and Maxwell (1972), who injected autogenous blood into the anterior chamber, and the processes they described are similar to those observed in the human meshwork. The erythrocyte is surrounded by cell processes and is incorporated within a vacuole in the cytoplasm of the macrophage; it appears that at least two mechanisms are involved in the subsequent breakdown of the erythrocyte. The electron dense laminated material within lysosomal complexes in the cytoplasm of the macrophages probably represents collapse and disintegration of the erythrocyte membrane-similar structures were described by Collet (1970) in his study of erythrocyte phagocytosis by pulmonary alveolar macrophages. Whether or not the haemoglobin is dealt with independently is not determined by this study, but the presence of intracytoplasmic aggregates of finely granular material surrounded by a membrane suggests that haemoglobin is degraded within the cell. Similar structures within lysosomal complexes of mouse spleen macrophages have been shown to contain iron by Daems, Cornelisse, and Poelmann (1972) using $x$-ray microanalysis of ultrathin sections. The breakdown products might be extruded from macrophages and the presence of electron dense bodies in the connective tissue beneath the lining endothelium implies that this might occur, but an apparent extrusion of intracytoplasmic debris was not encountered in this investigation and the electron-dense bodies could have been derived from red cells broken down by extracellular digestion or disintegration. The results of this investigation indicate that a significant method of erythrocyte disposal is by migration of debris-laden macrophages through the meshwork to Schlemm's canal and hence to the systemic reticulo-endothelial system. This adds support to the proposal put forward by Rohen and van der Zypen ( 1968 ).

It would appear from this and other investigations that macrophages (Vegge, i967; Segawa, I968; Rohen and van der Zypen, I968) and polymorphonuclear leucocytes (Hogan, Alvarado, and Weddell, I97I) can pass through the lining endothelium, either between cells or through the cytoplasm, but they may also utilize the giant vacuoles which are characteristic of these cells (Tripathi, I97I; Inomata, Bill, and Smelser, 1972). Although giant vacuoles have been shown to be involved in the transmission of particles, e.g. ferritin (Feeney and Wissig, 1966; Tripathi, 1971), pseudoexfoliation material (Ringvold and Vegge, I97I), and bacteria (Lee, I97I), there is no ultrastructural evidence 
from either the present study or that of Shabo and Maxwell (1972) that vacuolated endothelial cells are involved in the transmission of erythrocytes and their breakdown products. It is difficult to reconcile this failure to demonstrate transendothelial erythrocyte diapedesis with the elegant demonstrations by Hørven (1963) and Bill (I968), which showed, in humans and monkeys respectively, that intracameral ${ }^{51} \mathrm{Cr}$-labelled erythrocytes could be retrieved from the peripheral circulation as intact cells. Hørven (1964) also used autoradiography to confirm his investigations and referred to the general literature on diapedesis to support his hypothesis that erythrocytes can pass freely through the endothelium. Hørven's hypothesis has since been confirmed by morphological studies at the ultrastructural level in man (Kayes, 1967) and in monkeys following experimental hyphaema (Inomata and others, 1972), and Bill (1970) has demonstrated constricted erythrocytes in transit through the lining endothelium of Sclemm's canal by scanning electron microscopy. However, in this human material, we were dealing with a pathological process which had been present far longer than in the experimental situations described above. It is reasonable to expect, therefore, that the cellular reactions were modified and possibly that the diapedetic facility of erythrocytes was impaired, either by the presence of fibrin and other proteins or by an alteration in the extracellular ground substance.

Although the uveal and corneo-scleral parts of the meshwork showed advanced pathological changes, it is notable that the lining endothelium of the trabecular wall showed only occasional segments of focal damage and the widespread changes due to histamine release, e.g. contraction of cell cytoplasm and nuclear infolding (Majno, Shea, and Leventhal, 1969), were not in evidence. The presence of platelet thrombi (French, MacFarlane, and Sanders, 1964) on small foci of endothelial damage is of interest, and these may be the precursors of the fibrovascular organization seen in Schlemm's canal in many eyes enucleated some months after trauma.

From this investigation and that of other authors, we are now in a position to add information which clarifies the pathogenesis of haemolytic glaucoma (Fenton and Zimmerman, 1963). The endothelial cells are activated very soon after exposure to erythrocytes and the process of phagocytosis begins within 8 hours (Shabo and Maxwell, 1972). The earliest signs of increased activity are gaps between the trabecular endothelial cells, and the ultrastructural changes within the cytoplasm indicate an increased mobolic activity. The endothelial cells lose contact with each other and detach from the trabecular beams which, without the protective influence of the endothelial cells, become swollen and disrupted. If the demand for phagocytosis is excessive, histiocytic macrophages appear in large numbers and, together with the detached endothelial macrophages, they are washed or they migrate into the endothelial meshwork where some of them penetrate the cells of the endothelial lining to enter the general circulation. The accumulation of macrophages and erythrocytes in intertrabecular spaces narrowed by swollen trabecular beams is likely to obstruct outflow in the extreme case. This obstruction would be intensified by the fibrovascular organization which follows platelet thrombus formation on the damaged endothelial lining of Schlemm's canal.

\section{Summary}

Examination of the ultrastructure of the outflow apparatus of a 20-year-old man 15 days after a traumatic hyphaema has emphasized the role of phagocytosis as a mechanism of disposal of erythrocytes. Two types of cell were shown to be involved in erythrocyte 
phagocytosis: histiocytic macrophages and endothelial macrophages. The endothelial macrophages detached from the trabecular beams and migrated with the histiocytic macrophages to accumulate in the endothelial meshwork, and on several occasions macrophages were observed in transit through the cytoplasm of the endothelial lining of the trabecular wall of Schlemm's canal. The intercellular junctions of the lining endothelium were preserved and there was no evidence of transendothelial erythrocyte migration. The results help to clarify the pathogenic mechanisms involved in haemolytic glaucoma.

This work was, in part, supported by Grant No. 395 provided by the Scottish Hospital Endowments Research Trust. This aid is gratefully acknowledged. We also wish to thank Prof. W. S. Foulds for permission to use material from this case. For use of the electron microscope we are indebted to Prof. J. A. J. Pateman.

\section{References}

\begin{aligned} BILL, A. (1968) & Invest. Ophthal., 7, 156 \\ \hline (1970) & Exp. Eye Res., 10, 214\end{aligned}

GOLLET, A. J. (1970) Anat. Rec., 167, 277

Daems, W. TH., CORnelisse, c. J., and PoelmanN, R. E. (1972) In "Proc. Fifth Conference on Electron

Microscopy", p. 264. Institute of Physics, London

fEeney, L., and wissig, s. (ig66) Trans. Amer. Acad. Ophthal. Otolaryng., 70, 79 I

fenton, R. H., and zimmerman, L. E. (1963) Arch. Ophthal. (Chicago), 70, 236

french, J. e., macfarlane, R. G., and sanders, A. G. (1964) Brit. F. exp. Path., 45, 467

hogan, M. J., Alvarado, J. A., and weddell, J. E. (I97I) In "Histology of the Human Eye",

p. 149. Saunders, Philadelphia

HOLMBerg, Å. s. (1965) Docum. ophthal. (Den Haag), 19, 339

HøRVEN, I. (1963) Acta ophthal. (Kbh.), 4I, 402

- (1964) Ibid., 42, 600

inomata, H., Bill, A., and Smelser, G. K. (1972) Amer. F. Ophthal., 73, 760

IWAMOTO, T., WITMER, R., and LANDOLT, E. (I97I) Ibid., 72, 420

KAYES, J. (1967) Invest. Ophthal., 6, 38 I

LEE, W. R. (I97I) Trans. ophthal. Soc. U. K., 91, 687

majno, G., shea, s. m., and leventhal, M. (1969) J. Cell Biol., 42, 647

ringvold, A., and vegge, t. (197 I) Virchows Arch. Abt. A. Path. Anat., 353, i 10

rohen, J. W., and VAN Der ZYPEN, E. (I968) v. Graefes Arch. Ophthal., 175, 143

SEGAWA, K. (1968) Med. F. Shinshu Univ., 13, 57

Shabo, A. L., and MAXWell, D. s. (1972) Amer. F. Ophthal, 73, 25

TRIPATHI, R. C. (1969) Trans. ophthal. Soc. U.K., 89, 449

- (1971) Exp. Eye Res., I1, I1 6

(1972) Brit. F. Ophthal., 56, 157

VEGGE, T. (1967) Z. Zellforsch. mikrosk. Anat., 77, 427

weIss, L. (1972) In "The Cells and Tissues of the Immune System", p. 107. Prentice-Hall,

New Jersey, U.S.A.

yamashita, T., and Rosen, D. A. (1965) Amer. J. Ophthal., 60, 427 